\title{
Physics students' construction of differential length vectors in an unconventional spherical coordinate system
}

\author{
Benjamin P. Schermerhorn ${ }^{1, *}$ and John R. Thompson ${ }^{1,2}$ \\ ${ }^{1}$ Department of Physics \& Astronomy, University of Maine, Orono, Maine 04469, USA \\ ${ }^{2}$ Maine Center for Research in STEM Education, University of Maine, Orono, Maine 04469, USA
}

(Received 25 May 2018; published 14 February 2019)

\begin{abstract}
Vector calculus and multivariable coordinate systems play a large role in the understanding and calculation of much of the physics in upper-division electricity and magnetism. Differential vector elements represent one key mathematical piece of students' use of vector calculus. In an effort to examine students' understanding of non-Cartesian differential length elements, students in junior-level electricity and magnetism were interviewed in pairs and asked to construct a differential length vector for an unconventional spherical coordinate system. One aspect of this study identified symbolic forms invoked by students when building these vector expressions, some previously identified and some novel, given the vector calculus context. Analysis also highlighted several common ideas in students' concept images of a non-Cartesian differential length vector as they determined their expressions. As no interview initially resulted in the construction of an appropriate differential, analysis addresses the role of the evoked concept images and symbolic forms on students' performance.
\end{abstract}

DOI: 10.1103/PhysRevPhysEducRes.15.010111

\section{INTRODUCTION}

Mathematics often provides an underlying framework for the understanding and learning of physics. It can be used to describe physical scenarios or intertwined with physics knowledge for the purpose of reaching a deeper understanding. The ability to understand and use mathematics this way becomes increasingly relevant in upperdivision physics courses as more sophisticated mathematical concepts, equations, and expressions become ever present. As such, student understanding of mathematics in various areas of physics has become a recent topic of focus in physics education research [1-9]. At this level, the use of mathematics includes assessing underlying physical symmetry, interpreting physical situations for the purpose of calculation, and interpreting the results of calculation in terms of the given physical situation. These three areas of mathematics in particular have been cited as student difficulties in upper-division electricity and magnetism (E\&M) [5].

While the use of mathematics in upper-division E\&M requires students to understand and apply concepts from multiple domains, single-variable and multivariable calculus concepts are the most prevalent across the curriculum,

\footnotetext{
*benjamin.schermerhorn@maine.edu

Published by the American Physical Society under the terms of the Creative Commons Attribution 4.0 International license. Further distribution of this work must maintain attribution to the author(s) and the published article's title, journal citation, and DOI.
}

from standard integration through mathematical operations involving vector calculus and differential vector operators. The caveat with regard to vector calculus is that these mathematical conceptualizations need to be reasoned about with attention to symmetries specific to the physical situation (e.g., spherical coordinates for point charges and cylindrical coordinates for straight wires and lines of charge). When integrating, students are expected to construct and implement differential elements (i.e., lines, areas, and volumes) within these coordinate systems, as detailed by the nature of the electric or magnetic fields created by a charge or current distribution. In the majority of vector calculus problems, in which students are summing up the dot products of various vector fields over specific paths or surfaces, these differential elements have a particular direction and are represented by vectors. This method of treating differential elements in terms of their own coordinates differs greatly from mathematics treatment of vector calculus, which typically employs a Cartesian approach and deals with non-Cartesian paths or surfaces by parametrization [10].

Given the various distributions of charges and currents, the respective field symmetries in E\&M often dictate the use of a non-Cartesian coordinate system to simplify the vector calculus formalism. As such, the differential elements are represented differently and have different variables to dictate direction than the traditional $d x$ or $d y d z$, a line element and area element both in the $\hat{\mathbf{i}}$ direction.

In cylindrical and spherical coordinates, motion along curved surfaces results in differential length elements that 
are arc lengths and include scaling factors (e.g., $\overrightarrow{d l}_{\theta}=$ $r d \theta \hat{\boldsymbol{\theta}}$ for spherical coordinates in physics). Area elements are constructed by determining which variables are changing and which are constant, and then multiplying the differential length elements associated with the changes.

The cross product of $\overrightarrow{d l}_{\theta}=r d \theta \hat{\boldsymbol{\theta}}$ and $\overrightarrow{d l}_{\phi}=r \sin \phi d \phi \hat{\boldsymbol{\phi}}$ yields the differential area for the surface of a sphere, a differential area commonly used in Coulomb's and Gauss's laws. This type of problem solving appears early in a typical E\&M course text (see, e.g., Griffiths [11]). Therefore the differential length vector in each coordinate system emerges as a fundamental mathematical construct in the application of vector calculus in upper-division electricity and magnetism courses.

In order to investigate student use and understanding of non-Cartesian differential elements, course observations were conducted in junior-level E\&M, leading to the design and implementation of an interview protocol. These observations included detailed field notes and the collection of homework, quiz, and test data prior to grading. Preliminary review of exam data collected during course observations revealed that almost all students correctly wrote spherical differential area elements at the end of the first semester of a two-semester E\&M sequence, while only slightly more than half were able to express the area element for cylindrical coordinates. This contrasts with coordinate system performance on a quiz given as part of the course in the earlier portion of the semester, where more students were able to write a differential length vector for cylindrical coordinates than for spherical coordinates.

In an effort to elicit ideas during the construction of $\overrightarrow{d l}$, interviews were conducted in which students were asked to construct differential elements in an unconventional spherical coordinate system. This served to answer the following questions:

(1) To what extent do students understand the multivariable coordinate systems used in E\&M?

(2) What ways do students build and/or determine differential length elements in multivariable systems?

(3) What understanding do students have regarding the conceptual and geometric ideas and mathematical structures needed to construct a generic differential length vector?

To investigate students' understanding of the symbolic expressions and conceptual aspects of non-Cartesian differential length vectors, we applied two analytical approaches: symbolic forms [12] to investigate students' construction of the aspects of the differential length expression, and concept image [13] to elucidate aspects of student understanding pertaining to differential length vectors. The specifics of these frameworks are discussed further in Sec. III. The task and methodology are discussed in Sec. IV, and the results are presented in Sec. V.

\section{RELATED LITERATURE}

Understanding a differential vector element requires students to reconcile ideas related to differentials and the coordinate systems that shape the specifics of differential line, area, and volume elements. Historically, differential elements, integration, and coordinate systems have been individually identified as areas of difficulty for students.

Research exploring students understanding of differential areas in E\&M developed from a need for students to consider infinitesimal fields and charges. Hu and Rebello have identified several resources and conceptual metaphors for differentials related to students' understanding of these quantities as small amounts, unitless points, a cue to differentiate a formula to derive a second differential quantity, and as an indicator of the variable of integration [14]. The idea of a differential as a small amount is supported by work on integration in physics where students identify the need to add up "little chunks" using an integral $[2,15]$. Other work on integration identified a specific difficulty for students in determining the meaning of the differential area element as it appeared in an integral [16]. This work highlights the fact that many students often disregard the meaning of differential as a variable quantity when performing calculations. Students commonly see the differential as merely a variable of integration, with little physical meaning, even when integrating in physics. This represents a common finding in mathematics literature as well [8,16-18].

Within the realm of vector calculus, physics education researchers have identified difficulties in student understanding when applying Gauss's and Ampère's laws [3,5,19-21]. These equations are constrained to highly symmetric situations. Thus while these laws involve a surface integral and line integral, respectively, much of this work focuses on how students recognize and apply specific symmetries. While detailing student difficulties with mathematics in E\&M, Pepper and colleagues noted two instances in a homework help session where students had difficulties connecting coordinate systems to differential elements [5]. In one case, students neglected to include the necessary scaling factors when writing spherical differential areas, using $d a=d \theta d \phi$, rather than $d a=r^{2} \sin \theta d \theta d \phi$. Another group attempted a line integral in three dimensions using $d x d y d z$ as a length element. These types of errors are consistent with both a difficulty accounting for the underlying physical system and a limitation in students' understanding of how to construct and apply differential elements.

One possible explanation for students' use of $d a=$ $d \theta d \phi$ is that it results from an attempt to match the patterns of differential elements as written in Cartesian coordinates. Hinrichs found this to be the case when asking students to construct $\vec{r}$ in spherical coordinates for several points laying along the Cartesian axes [22]. In these cases students provided answers with three components, rather than the 
correct $r \hat{\mathbf{r}}$. Students here also had difficulty correctly expressing the conventional physics values of $\theta$ and $\phi$, often reversing the notation for the angles to align with typical mathematics representation. Work in sophomorelevel mechanics that addressed student understanding of coordinate systems supports students' favoring of Cartesian, and has found students are less comfortable with applying polar coordinates [23]. Other literature has also shown students difficulty articulating the conventions of non-Cartesian coordinate systems when defining unit vectors in polar coordinates [24].

Research into student understanding of multivariable coordinate systems is a relatively recent venture in undergraduate mathematics education research. However, some literature has explored students reasoning about covariational relationships when plotting graphs in polar coordinates, and found that students often try to translate graph and function meanings that were taught in Cartesian coordinates, despite these meanings becoming inappropriate with changes in representation (i.e., the vertical line test) [25]. This supports the idea that the development of student understanding purely in Cartesian coordinates makes it difficult for students to develop understanding of nonCartesian coordinate systems.

Despite significant forays into various levels of mathematical understanding, little work has explored student understanding of the differential vector element, in particular how it is constructed in the non-Cartesian systems commonly used in associated physics problems. This work seeks to take a step toward analysis of student understanding of the differential vector elements as they appear in the non-Cartesian coordinate systems of E\&M, specifically the differential length vector.

\section{THEORETICAL PERSPECTIVES}

To analyze student construction of the differential vector element as a dynamic process, we employ two complementary analytical frameworks: symbolic forms [12] and concept image [13]. Concept images gives insight into what particular ideas students evoked during construction of a differential vector element, while symbolic forms identifies the specific structures students created during construction and how they relate to student thinking.

\section{A. Symbolic forms}

The symbolic forms framework [12] was developed based on the knowledge-in-pieces model [26] and identifies the structural components of equations based on students constructing and interpreting expressions. Sherin's initial study involved students in a third-semester introductory physics course, in which students were provided with several word problems modeling physical situations common to introductory physics. The equations students constructed for given situations involved scalar quantities and the mathematics was limited to basic algebraic manipulation. The development of a symbolic forms analysis provided a critical lens for the investigation of students' construction and sense-making of equations at the introductory level.

The specific nature of a symbolic form comes from the combination of a symbol template with a conceptual schema. A symbol template is an externalized structure of an equation. One example of a symbol template is $\square+\square+\square$; each box represents one or more variables and/or numbers, depending on what a student deems fit. A student's conceptual schema is the intuitive internalized mathematical idea that the student expresses in the template. Sherin defines the conceptual schema as simple structures, similar to phenomenological primitives [26]. Furthermore, these schema can vary for the same mathematical operation. One reason to add quantities is when the sum represents a whole quantity and each term in the addition-each box-represents one component of that quantity. For example, in the expression for the surface area of a cylinder of radius $R$ and length $L$, there is an area term for the side $(2 \pi R L)$ and a term for the (two) ends $\left(2 \pi R^{2}\right)$. The symbolic form associated with this particular templateschema pair is known as parts of a whole [12].

Sherin further identified different means by which students invokes addition. A student could interpret the expression $v_{o}+a t$ as an initial velocity quantity plus some increase or decrease depending on the acceleration. The schema behind this kind of addition is identified as base + change, and has the associated template $\square+\Delta$ [12]. The box represents the initial quantity, while a triangle (presumably referring to the symbol for the Greek letter delta) accounts for the term that causes the change.

Overall, the conceptual schema is what informs how students need to write particular expressions and accounts for their understanding of the template. The symbol template is then the manifestation of the conceptual schemata as a reified symbolic pattern. Symbolic forms analysis lives almost entirely in the structural realm of the equations; as defined by Sherin, the conceptual schema is conceptual in the sense of justifying the mathematical operation, but not the conceptual understanding of the physical scenario that leads to it. In other words, symbolic forms were not developed to interpret student understanding of the physics represented by a particular equation. In the knowledge-in-pieces tradition, the correctness of the equation in terms of physics is irrelevant; the focus is on the structure.

Work with symbolic forms has expanded into the upper division and has explored different kinds of integration in mathematics. Meredith and Marrongelle [15] adapted the conceptual aspects of symbolic forms to describe what features of electrostatics problems cue students to integrate. They found students using the dependence form, which necessitates the need for and inclusion of a particular 
variable that the expressions "depends on," when eliciting the reliance of an integration on a particular variable. Students were also found to invoke the parts of a whole form when summing up multiple small charges. The ideas of symbolic forms were additionally used to analyze calculus students' ideas when making sense of integrals; the identified conceptual schema often included graphical representations of given functions [18]. The symbolic forms framework has been used to analyze physical chemistry students' use of partial derivatives in thermodynamics [27]. This work further showed how recall can play a role in mediating student construction in the upper division.

We use the symbolic forms framework to provide insight into how upper-division students develop the structure of differential vector elements and determine how each component is represented in the final equation. Additionally, a symbolic forms approach provides a lens to interpret student understanding as they read information out of an equation, such as relationships between quantities, by interpreting the templates used by an equation.

\section{B. Concept image}

A strict symbolic forms analysis neglects the content basis for choices, using only procedurally based mathematical justifications for the symbolic arrangements which indicate only that a student needs a particular structure in their expression. Thus a framework that includes student thinking about the content knowledge that goes into choosing particular forms was necessary. Given the mathematical nature of this work, we imported the concept image framework [13] as an independent analytical lens from research in undergraduate mathematics education to round out our investigation of students' construction of the differential length element.

A student's concept image is a multifaceted understanding that represents that student's entire cognitive structure about a particular idea, including any properties, processes, and mental pictures a student may recall. Unlike the actual concept definition, which is what is provided by textbooks and formal theorems, the concept image is a dynamic construct dependent upon specific contexts and may contain elements that are contradictory or false. The idea of concept image has recently been adopted by physics education researchers studying student use of mathematics, particularly in the context of vector differential operators and integration in electromagnetism courses $[1,2]$.

By analyzing the aspects of the concept image that are elicited by the students within a specific context, known as the evoked concept image, researchers can gain specific insights about how students think about that concept. For example, integration may elicit several evoked concept images, such as Riemann sum or area under the curve [2], depending on the task being administered. While students may possess other ideas related to integration, knowing whether the students' evoked concept image is something reminiscent of Riemann sums or area under the curve tells one how students interpret particular problems. Likewise, it is also telling if the student only grasps integration in terms of procedure, e.g., that the integral of $n x^{n-1} d x$ is $x^{n}+C$, without being able to recall what that means. For our work, cataloging responses from students' construction of differential vector elements yields a set of ideas that comprise an evoked concept image in that context.

We combine the two frameworks described above in our work on student understanding of non-Cartesian differential elements. By incorporating the concept image framework, the symbolic forms analysis gains content meaning, which is not addressed by assessing students' conceptual schemata.

\section{RESEARCH DESIGN AND METHODOLOGY}

In order to investigate student understanding of how differential vector elements are constructed in nonCartesian coordinate systems, we developed an unconventional spherical coordinate system (Fig. 1). Students were shown the unconventional system in an interview setting and then were given a series of questions and tasks shown below:

(1) Does this depict a feasible coordinate system and if it is valid, what type of situations (kinds of problems) would it be appropriate for?
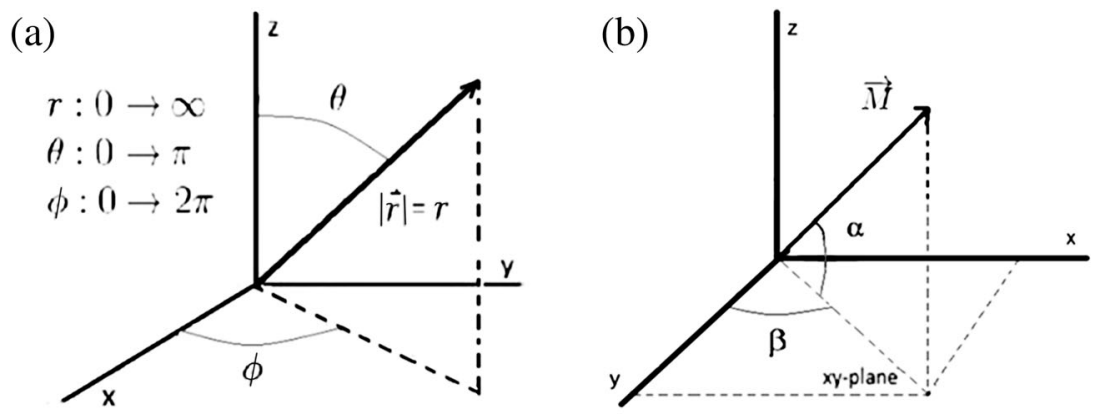

(c)

$$
\begin{aligned}
& \overrightarrow{d l}=d r \hat{r}+r d \theta \hat{\theta}+r \sin \theta d \phi \hat{\phi} \\
& d \tau=r^{2} \cos \theta d r d \theta d \phi
\end{aligned}
$$

(d)

$$
\begin{aligned}
\overrightarrow{d l} & =d M \hat{M}+M d \alpha \hat{\alpha}+M \cos \alpha d \beta \hat{\beta} \\
d \tau & =M^{2} \cos \alpha d M d \alpha d \beta
\end{aligned}
$$

FIG. 1. (a) Conventional (physics) spherical coordinates; (b) an unconventional spherical coordinate system given to students, for which they were to construct differential length and volume elements. The correct elements for each system are shown in (c) and (d), respectively. 
(2) Construct a differential length element, $\overrightarrow{d l}$, for the system.

(3) Construct a differential volume element for this coordinate system.

(4) Check that the volume element is correct.

By developing an unconventional coordinate system, we could determine students' abilities to work with the underlying conceptual ideas associated with the construction of these terms, rather than their ability to recall a memorized answer.

The unconventional system, which we call "schmerical coordinates," is left handed and depicts both angles as measured from the $y$ axis. The left-handed nature allows us to determine if any Cartesian elements presented by students are the result of recall or accurate (but unnecessary) projections, but has no bearing on the differential length element. This also means that the polar angle $\beta$ is placed differently than the analogous $\phi$ in spherical coordinates while not changing the length element. Likewise, the placement of the azimuthal angle $\alpha$ is different than that of $\theta$. The variation in the placement of the angles results in a $\cos \alpha$ in the differential length element, in contrast to the $\sin \theta$ in spherical coordinates. These deviations from spherical coordinates require students to attend to the ideas of building differential elements.

We focus our analysis here on the construction of the differential length vector, before students were asked to construct and check the differential volume element.

Clinical think-aloud interviews were conducted at two (public) universities with students enrolled in junior-level $\mathrm{E} \& \mathrm{M}$, which is a two-semester sequence at both institutions. Notably, this task was novel for students at both universities, since neither E\&M course incorporates unconventional coordinate systems. Four pairs of students $(N=8)$ were interviewed at university $\mathrm{A}$ at the end of the first semester and two pairs and a single student $(N=5)$ at university $\mathrm{B}$ at the beginning of the second semester of E\&M. Students were selected on a volunteer basis and represented a mixture of low to high achievers. Pairs were formed based solely on students' availability. Pair interviews facilitated student to student discussion and allowed them access to each other's conceptual understanding, thus minimizing the input and influence of the interviewer. For brevity, groups are identified in lists as $\mathrm{AB}$, $\mathrm{CD}, \mathrm{EF}$, and $\mathrm{GH}$ for the first university and PQ, RS, and T for the second. These identifiers signify pairings of students Adam and Bart (AB), Carol and Dan (CD), etc.

Each interview was videotaped and transcribed; transcriptions were analyzed to compile elements of students' evoked concept images of the differential length vector. Initial coding was completed by the first author with the purpose of identifying elements that appeared commonly across most groups. Samples from each category were checked by the second author and recoded when necessary. Any disagreement was discussed until consensus was reached. Concept image aspects were determined such that they did not share overlapping definitions and were not able to be broken down into multiple ideas. Once the aspects of the concept image were agreed upon, the data were recoded and checked between authors for agreement. Similarly, students' writing was analyzed throughout the stages of construction to identify uniform templates that might be connected to symbolic forms, either those identified by Sherin or new forms specific to this context.

A second independent analysis of evoked concept images in the unconventional coordinate system allows us to develop a clearer picture of student understanding, as well as to identify specific student difficulties [28] and successes when working with coordinate systems that they apply to particular problems throughout the semester.

\section{RESULTS AND DISCUSSION}

No group was able to construct a correct differential length element on their first attempt. Three pairs of students explicitly discussed their construction during the interview, elaborating on their choices of how they structured the equation and their inclusion or exclusion of certain terms, while others reasoned by recalling information from other coordinate systems. The majority of the data corpus presented here primarily comes from three groups at university A who constructed their differential length using and explaining particular ideas, due to the theoretical frameworks' focus on analysis of construction and student ideas. Students at university B had more significant difficulty reasoning about construction of the differential length vector, despite being able to connect the unfamiliar system with spherical coordinates. One of these groups, PQ, as well as the fourth group at the first university, $\mathrm{GH}$, relied heavily on recall and ended up working within the structure of a Cartesian differential length. The other two groups had difficulty accessing ideas of arc length or recognizing multiple components. However, common elements of reasoning did appear during portions of their construction, and thus these interviews provide additional supporting data to the existence of particular concept images and symbolic forms.

Analysis of student construction revealed several emergent symbolic forms (Table I). Some of the invoked symbolic forms were consistent with forms previously identified at the introductory level: parts of a whole, coefficient, and no dependence [12]. Additionally, we identified other forms that represented template-schema pairings not yet addressed from a symbolic forms perspective: magnitude direction and differential. The newly identified symbolic forms account for the increase in mathematical sophistication due to the need to express vectors and calculus concepts that were absent from the introductory-level problems given in the original literature.

In this section, we isolate students' invocation of symbolic forms into two generalized parts of construction. In the beginning of construction, most groups attended to 
TABLE I. Existing and novel symbolic forms identified in students' construction of a differential length element.

\begin{tabular}{lcc}
\hline \hline Symbolic form & Symbol template & Conceptual schema \\
\hline Parts of a whole & $\square$ No dependence & $\begin{array}{l}\text { Accounts for multiple components that contribute to a larger hole [12]. } \\
\text { Indicates an expression is independent of, or not a function of, a } \\
\text { specific variable [12]. } \\
\text { Represents a quantity seen as just a number or a constant (possibly } \\
\text { having units) put in front of an expression [12]. }\end{array}$ \\
Magnitude direction & $\begin{array}{c}\text { Used to denote a vector expression including the magnitude of a } \\
\text { quantity (having units) and a unit vector to indicate a specific } \\
\text { direction. } \\
\text { Representation of a differential quantity. Typically invoked here as a } \\
\text { small amount of or infinitesimal change in a quantity. }\end{array}$ \\
\hline \hline
\end{tabular}

the vector or component nature of the differential length element. At this stage groups constructed templates consistent with parts of a whole and magnitude direction forms. Subsequently, groups typically discussed the structure of each component, attending to the ideas related to the magnitudes of each component, which involved developing the templates associated with the differential, coefficient, and no dependence symbolic forms.

At various stages students' concept images motivated the need for various symbolic forms, as well as helped students determine the particular variables needed to complete construction (Table II). Analysis has identified four aspects of student's concept images associated with the properties of a non-Cartesian differential length vector: component and direction, dimensionality, differential, and projection.
Similarly, three processes were also identified across student work that assisted construction: grouping of like terms, rote recall, and transliteration (Table III). The elements of the concept image do not provide an exhaustive description of the ideas or processes appearing during construction, but instead represent a list of elements common to more than one group. Notably, each of these elements appeared in four to six of the seven interviews.

\section{A. Elements related to vector properties}

At the outset of construction, five of the student groups attended to the component and direction aspect of differential length elements, highlighting the need for a summation of three different components. Groups GH and PQ

TABLE II. Aspects of students' concept image of a differential length vector in a non-Cartesian coordinate system.

\begin{tabular}{|c|c|c|}
\hline Concept image aspect & Associated idea & Example (in bold) \\
\hline Component and direction & $\begin{array}{l}\text { Recognition of multiple components, } \\
\text { each displaced independently }\end{array}$ & $\begin{array}{l}\text { Frank: Yeah, so like there, } d l \text {, there are three different } \\
d l \text { 's. There is } d l \text { with respect to } M, d l \text { with respect to } \\
\text { a, } \alpha \text {, and } d l \text { with respect to } \beta \ldots\end{array}$ \\
\hline Dimensionality & Each term needs units of length. & $\begin{array}{l}\text { Adam:... This doesn't have any units of length ... so, it } \\
\text { needs to have some } M \text { term. }\end{array}$ \\
\hline Differential & $\begin{array}{l}\text { Small changes or amounts (of } \\
\text { displacements) }\end{array}$ & $\begin{array}{l}\text { Carol: Right. So you have a change in your } \hat{\mathbf{M}} \text { is going to } \\
\text { be your } d M \text {, it's your change in your } M \text {. }\end{array}$ \\
\hline Projection & $\begin{array}{l}\text { Use of cosine or sine explicitly (not } \\
\text { rote recall) }\end{array}$ & $\begin{array}{l}\text { Elliot: ... but if we're pointed way up here, then we need } \\
\text { to take the cosine so that we're, we have the } \\
\text { component of } r \text { that is actually in the } \beta \text { plane,... }\end{array}$ \\
\hline
\end{tabular}

TABLE III. Actions taken by students during construction of a differential length vector for schmerical coordinates.

\begin{tabular}{|c|c|c|}
\hline Construction action & Associated idea & Example \\
\hline Grouping & $\begin{array}{l}\text { Combining elements by like variables or } \\
\text { terms. }\end{array}$ & $\begin{array}{l}\text { Harold: You've got } r d r \hat{\mathbf{r}} \text { plus, is it } \sin \theta d \theta \text { or is there an } r \\
\text { in there? }\end{array}$ \\
\hline Rote recall & $\begin{array}{l}\text { Writing or remembering elements from } \\
\text { Cartesian or spherical coordinate } \\
\text { systems. }\end{array}$ & Greg: $d \tau$ in spherical is $r^{2} \sin \theta=\ldots=d \theta d r=\ldots=d \phi$ \\
\hline Transliteration & $\begin{array}{l}\text { Direct matching of variables from } \\
\text { existing coordinate system. }\end{array}$ & $\begin{array}{l}\text { Bart: ... so now we have just to compare so we have } r \text { it is } \\
\qquad M, \theta \text { is } \alpha=\ldots=\phi \text { is } \beta \text {. }\end{array}$ \\
\hline
\end{tabular}


began their construction in Cartesian, while $\mathrm{AB}, \mathrm{CD}$, and EF attended to construction in schmerical coordinates, addressing that each component of the vector equation is an independent displacement of the vector $M$ in each of the variable directions. In each group, the component and direction aspect manifested as a combination of two symbolic forms: parts of a whole [12], which accounts for the inclusion of multiple terms, and magnitude direction, which expressed the direction associated with each component term.

\section{Parts of a whole}

The need for multiple components to completely express a differential length vector resulted in the invocation of the parts of a whole symbolic form by almost all groups. Frank demonstrated a requisite conceptual schema when starting construction:

Frank: There are three different dl's. There is $d l$ with respect to $M, d l$ with respect to $\alpha$ and $d l$ with respect to $\beta$.

Elliot: You sum them, so $[\overrightarrow{d l}]$ is those added together: $d M \hat{\mathbf{M}}+M d \beta \hat{\boldsymbol{\beta}}+M d \alpha \hat{\boldsymbol{\alpha}}$.

Elliot and Frank worked on each component independently; Elliot then summed these components to express their full (incorrect) vector differential at the end of their construction. Adam and Bart constructed their differential element in a similar manner. Carol and Dan started with the overall structure, accounting for the unit vector of each component, and subsequently filled in each term (Fig. 2). Each of these groups recognized the need for and express the multiple components associated with the differential vector element in this coordinate system. The expression of multiple terms with the conceptual schema of "three different $d l$ 's" that must be summed or "added together" makes this consistent with Sherin's parts of a whole symbolic form.

Perry and Quinn recognized the need to sum multiple components but were unable to disentangle themselves from Cartesian coordinates. They initially structured their differential length as the addition of three $d l$ 's for each Cartesian direction (Fig. 3), invoking the parts of a whole template but for the incorrect coordinate system.

Rachel and Silas, having first decided that $\overrightarrow{d M}$ was sufficient to describe the differential length element, later
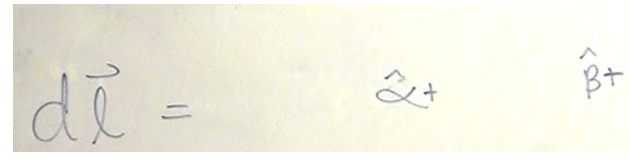

FIG. 2. Beginning stages of construction for Carol and Dan showing the coupling of the parts of a whole and magnitude direction symbolic forms.

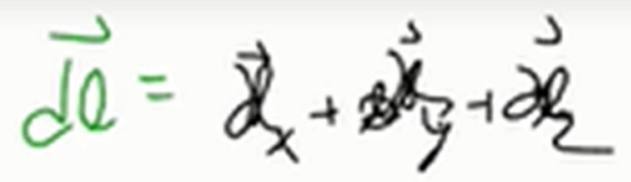

FIG. 3. Perry's and Quinn's writing of the differential length elements as a sum of three terms, depicting the parts of a whole structure despite writing out each component explicitly.

\section{$d l=\langle d M, d \alpha, d \beta\rangle$}

FIG. 4. Rachel's and Silas's final expression for their differential length vector.

remembered having also used $d l$ as a description of circular paths and recognized the need for multiple terms:

Rachel: ... it's like a path along something, so like that is fine if the path is like in the $d M$ direction but if it is not then [dl] is not very generic... there would have to be three components to it... because it has three dimensions.

Rachel and Silas then represent this new $d l$ using bracket notation for vectors (Fig. 4). While the group encodes their length vector using this bracket vector notation, their conceptual schema is illustrative of parts of a whole and explicitly explains students' summation of only three terms. Following construction of the template for the full differential length element, several groups attended explicitly to the dimensionality of each component.

Carol: ... and then they each need to be a length.

Elliot: ... and each of them need to be a length.

This need for dimensionality became increasing relevant when students made decisions about what terms belonged in each component, but was recognized early on in construction.

\section{Magnitude direction}

Either following or coupled with the parts of a whole symbolic form, students' attended to the vector nature of components. Students split each component into a pair of two distinct parts, one that displays the magnitude of the differential length term, and the other the direction each component is associated with. We identify this particular product as the magnitude direction symbolic form with the template $\square \hat{\square}$. The work of Carol and Dan displays this explicitly, as they left space to write the magnitudes of the 


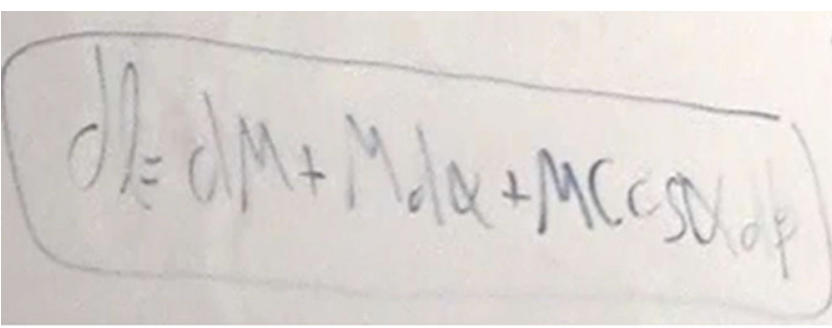

(a)

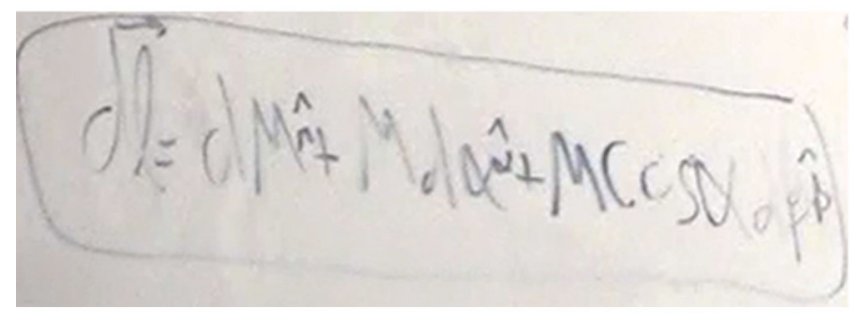

(b)

FIG. 5. Greg and Harold before (a) and after (b) recognizing the need to include unit vectors.

components in the expression (Fig. 2). During a second attempt to construct a differential length element motivated entirely by rote recall, GH completed their expression by adding a unit vector to each of the summed differential length magnitudes (Fig. 5).

While some students inherently included the vector nature when constructing components, other students paid particular attention to the unit vector of the component, using it to reason about the preceding magnitude in that direction.

Carol: So, dl is like you just have some path. So I'm trying to think, like, if I was going to walk in the $\hat{\boldsymbol{\alpha}}$ direction...

Elliot: So you're going to have a length component in the $\hat{\boldsymbol{\beta}}$ direction.

Each student here isolates the specific direction or unit vector and then attends to the magnitude of the component as a second entity. After reasoning about the nature of what is included in the magnitude of the component, students automatically write the magnitude of the vector component as preceding the unit vector as it is typically expressed in physics.

\section{B. Elements related to construction of the magnitude of components}

After developing the overall structure for the equation, groups attended to the individual components, accessing various concept image aspects to fill the magnitude direction template. Most specifically, this involved a combination of Sherin's coefficient and the newly labeled differential symbolic forms. While the differential symbolic form involved reasoning about small changes and infinitesimally sized quantities, the coefficient form had more varied conceptual schemata involving attention to dimensionality and geometrical reasoning, rote recall and mapping. Several students also invoked the no dependence symbolic form to distinguish which variables depend upon the others in the coordinate system (i.e., the arc length in the $\hat{\boldsymbol{\beta}}$ direction being dependent on the angle alpha).

\section{Differential}

In addition to its identification as a symbolic form, differential appeared as an aspect of students' evoked concept image, depicting student attention to needing small displacements or small changes in specific directions, as seen in the following excerpts:

Carol: Right. So you have a change in your $\hat{\mathbf{M}}$ is going to be your dM, it's your change in your $M$.

Rachel: Um, $[d \vec{M}]$ represents a tiny portion of like, a length, or a change in the radial component of the vector.

Given the importance of the differential and the distinct attached conceptual schemata, we identify the differential symbolic form, $d \square$, from students' work. The form itself is similar to what appears in graphically oriented symbolic forms for integration, where students describe $d x$ as a "small portion of each graph," width of rectangle in a sum, a specific shape depending on the shape of the function (e.g., circle or square), or commonly just a cue for integration [18]. For students constructing differential vector elements, the differential is not (yet) associated with a particular integral expression, and thus is treated as a stand alone quantity with its own attached schemata as a need to represent a small quantity. When removed from the context of integration, there are a number of other conceptual ideas attached to differentials, especially in E\&M [14]. The treatment of differentials in terms of small amounts of motion or changes of a quantity was helpful to the building of terms and have been identified as productive to physics thinking in previous literature $[7,14,17,29]$. The ideas of the differential as a small amount or a change is trivial for students here, but other views also lead to the representation of the differential element.

Tyler expressed ideas consistent with the conceptual metaphor of the differential as a machine [14], which cued him to perform a differentiation. He initially represented $\vec{r}$ as a pattern-matched form of a vector in Cartesian coordinates [22], then attempted to determine partial derivatives from particular components.

Tyler: So any vector $r$, thats an $M \hat{\mathbf{M}}+\alpha \hat{\boldsymbol{\alpha}}+\beta \hat{\boldsymbol{\beta}}$...so is, I mean/ we're not looking for like the total $d r$ but like $d r / d M$ ? 


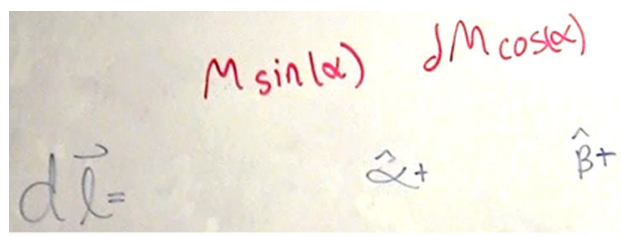

(a)

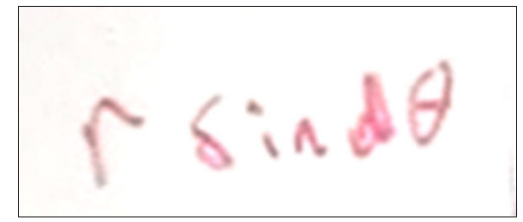

(b)

FIG. 6. (a) Carol and Dan incorrectly incorporating the idea of a differential by writing " $d$ " before their $\hat{\boldsymbol{\beta}}$ term. (b) Frank attempting to account for the arc length of a small angle and forcibly insert both a differential and trigonometric function into his expression.

With particular difficulty, Tyler begins to express this as $d \vec{r}=M d M+\beta$, explaining his $d \hat{\mathbf{M}}$ as a need to take the derivative of the unit vector to account for any "phase or time dependence." After being assured there was no time or phase dependence, he attempted to recall to specific coordinate transformations between spherical and Cartesian coordinate systems.

In some cases, difficulty reasoning about how to incorporate the differential led to students forcefully trying to insert a differential into their expressions. Other groups initially tried to forcefully insert a differential into their expression. After recognizing that $M \cos \alpha$ was a projection into the $x y$ plane, Carol and Dan wrote a " $d$ " in front of the whole expression [Fig. 6(b)]. Soon after, they label this an incorrect expression, they turned to recall of spherical coordinates. Similarly, Frank tried to express an infinitesimal arc length as $r \sin (d \theta)$ as a way to also explain where the differential and trigonometric function would appear [Fig. 6(b)]. After fixing this, group EF focused their construction on having a differential length component in a particular direction containing a differential with that variable:

Frank: So then you have $\beta$.

Elliot: $d \beta$.

Frank: Oh, yeah.

Elliot: So you're going to have a length component in the $\hat{\boldsymbol{\beta}}$ direction... so, basically we're going to need... an $M$... so it's $M$ times some $\Delta$, I think it's $M$ times $\Delta \beta, a$ small $\beta$, because it's like if you take $r$ times its small $\theta$ then that is the arc length (Fig. 7).

Elliot and Frank finally articulate this as the length component, $\overrightarrow{d l}_{\beta}=M d \beta \hat{\boldsymbol{\beta}}$, which now only lacked the needed trigonometric term, but correctly connects the expression of $d \beta$ with needing a small change in the angle.

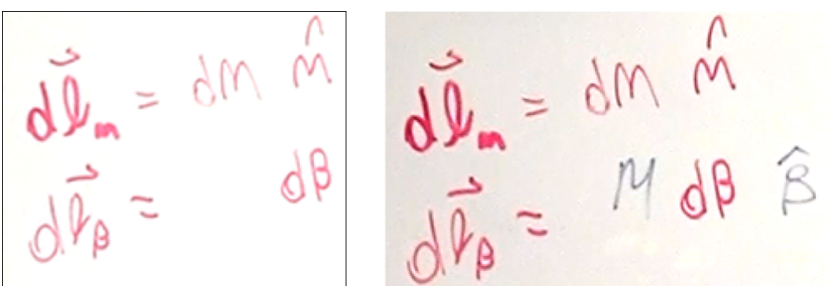

FIG. 7. Elliot and Frank constructing the $\beta$ component of the differential length. Initially they leave space to write the needed coefficient and unit vector. After discussion they include a coefficient lacking the projection term $\cos \alpha$.

Elliot and Frank's discussion here highlights another aspect of students' attention to the differential that ties into the magnitude direction symbolic form. As part of students' conceptual schema during construction, students eventually used the same variable from the differential symbolic form $(M, \alpha$, or $\beta)$ as the variable corresponding to the unit vector (i.e., $d M \hat{\mathbf{M}}, d \alpha \hat{\boldsymbol{\alpha}}$, and $d \beta \hat{\boldsymbol{\beta}}$ ). Greg and Harold do this inherently as they attend to the magnitude direction symbolic form (Fig. 5), while Carol and Dan explicitly recognize the need for pairing this after correcting a grouping error in a recalled spherical volume element. Students initially combined the $r \sin \theta$ with the $d \theta$ term, resulting with an $\alpha$ magnitude of $M \cos \alpha d \alpha$. After recognizing this mistake, they first switched only the differentials for the terms before recognizing the unit vectors would need to be switched as well, in order to keep the $d \alpha$ term with the $\hat{\boldsymbol{\alpha}}$ component.

\section{Coefficient}

The appearance of the coefficient symbolic form as a prefix to the differential form was most often predicated by the need for appropriate dimensions, recognition of arc length, or some level of rote recall to the more familiar spherical coordinate system. The coefficient form is generally invoked to include a space for specific factors or constants that appear in typical physics equations [12]. Students will often treat coefficients as parameters that "define circumstances under which [physics] is occurring" (p. 517). The symbolic form manifests physically in the equation as a term multiplied on the far left of a product of terms.

The most prominent and prescient evoked concept image was the need to include dimensionality, as seen in the following two (independent) excerpts:

Adam: This doesn't have any units of length, so it needs to have some $M$ term. (Fig. 8).

Carol: So, if it's going to be some trig thing but sine of something isn't a length so we're going to have to also have something else in there.

Students accessing of the dimensionality concept image aspect, both for the coefficient symbolic form and when 


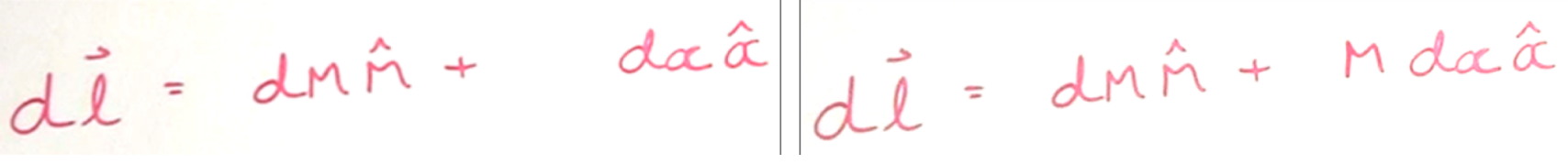

FIG. 8. Adam before and after using reasoning about including dimensionality to fill the coefficient template of the $\hat{\boldsymbol{\alpha}}$ component.

discussing the magnitude portion of their components, resulted in the inclusion of a $M$ or $d M$ term. With the $d M$ term satisfying the dimensionality, differential, and component and direction aspects, students did not include any more terms in the $\hat{\mathbf{M}}$ component. Elliot and Frank were the only group to engage in geometric reasoning and relate arc length as the actual physical justification for the $M$ and $M \cos \alpha$ in the $\hat{\boldsymbol{\alpha}}$ and $\hat{\boldsymbol{\beta}}$ components, respectively.

Elliot: Just like when you get the circumference, it's equal to $2 \pi r$, well it's taking all of the radians, instead [you take] a tiny amount of radians, which would give you a tiny arc length.

Elliot and Frank then construct the two angular components, but fail to recognize that the radius for the second arc length is a projection of $M$ in the $x y$ plane and end up with $\overrightarrow{d l}_{\beta}=M d \beta \hat{\boldsymbol{\beta}}$ as shown above (Fig. 7). $M$ is the important dynamic variable that the $\hat{\mathbf{M}}$ component depends on, but for the angle components where only the angles are changing it is a static variable representing a radius in an arc length.

Rachel and Silas do show express elements of geometric reasoning when constructing arc lengths to describe the sides of a differential volume as $M, M d \beta$, and $M d \alpha$, but the coefficient symbolic form was never cued in their final differential length (Fig. 4). They make no attempt at reconciliation between the two differential elements, and fail to recognize the need to do such. This is most likely due to difficulty with coordinate system understanding.

Rachel: I think it would be like, the first if it's in $r$ would be dr. Right? So you want it in Cartesian or in spherical?

Interviewer: I want it for this coordinate system.

Rachel: So I think $d l$ is just $d M, d \alpha, d \beta$, like commas in between those because that is how you figure out path... you have your change in your $M$ direction, then you have your change in your $[\alpha]$ direction...

The expression of $\overrightarrow{d l}$ as $<d M, d \alpha, d \beta>$ is sufficient for them as it accounts for the change in each direction. It is likely the students are attempting to map to a Cartesian representation of a differential length element, thinking that differential lengths expressed in their own coordinate systems look like the collection of differentials.

Rote recall and transliteration often occurred when students faced difficulties with the application of concept images or when geometric ideas were inaccessible. This is reminiscent of symbolic forms analysis of physical chemistry students construction of partial derivative expressions in the context of thermodynamics [27]. In these cases, recall mediated students' construction of equations in terms of particular processes, such as taking the total derivative, or as recall of specific concepts, such as $d X=0$ if $X$ is a constant.

While Elliot and Frank chose to not use a recalled to spherical differential element and focus construction specifically within the given schmerical system (with subsequent lack of attention to the projection aspect), groups $\mathrm{AB}$ and $\mathrm{CD}$ incorrectly included a $\sin (\theta)$ due to heavy reliance on spherical coordinates to complete the differential length vector. While Adam and Bart included the trigonometric function with little discussion, Carol and Dan discussed the origin of the components in spherical components after incorrectly grouping $\sin (\theta)$ with $d \theta$ in a recalled volume element.

Dan: ... You drop a $\sin (\theta)$ and then you're in $d \phi \ldots$ if I want to get down to $\phi$, I have to go down some $\theta$ or in this case $\alpha$ and then I rotate around and that is giving me my $\hat{\boldsymbol{\phi}}$ direction.

Despite recognizing the trigonometric function exists as a way of "getting down" to $\phi$ and thus belongs in the $\hat{\boldsymbol{\phi}}$ component, Dan failed to adjust the function for the change in angle, not making the final step in the writing of his differential length. Dan's interjection here served only to connect $\sin (\theta)$ with $d \phi$ and thus $\sin (\alpha)$ with $d \beta$. Dan's and Carol's focus on mapping between spherical and schmerical elements obscured the distinction between how sine and cosine act as geometric projections. The incorrect inclusion of sine in the schmerical length element is made to structurally match the coefficient space of the spherical element, rather than match the conceptual building aspect.

After initial difficulty with construction, Tyler decides "length is really only the radial component," and expressed $\overrightarrow{d l}$ as $M d M$ :

Tyler: ...Yeah, because it's the amount of $M$ for every little dM that I move... It's so much easier in Cartesian... but I think the only reason the $M$ is there is because when you transform coordinate systems, your length is no longer just $d M$.

Tyler then justifies his extraneous invocation of the coefficient form by citing the scaling factors gained by the spherical volume element when making the transformation from Cartesian coordinates. 
In many cases the coefficient symbolic form appeared as a means to complete an expression, driven most often by what Carol expressed as a "need to have something else in there." To accommodate for filling this space, students left blank spaces in the equation as if calling forth a particular template to fill in later. Specifically we see this for AB's (Fig. 8) and EF's (Fig. 7) inclusion of $M$ as the coefficient, but also earlier with CD (Fig. 2) as they sections out the necessary components, when invoking the parts of a whole symbolic form.

\section{No dependence}

The no dependence symbolic form appears when students explicitly address the absence of a variable in an expression. The need to project the vector $M$ into the plane of $\beta$ to get the requisite arc length results in the $\hat{\boldsymbol{\beta}}$ component being a function of the angle $\alpha$. In comparison, the arc length of the $\hat{\boldsymbol{\alpha}}$ component uses the full radius $M$ and ignores the coordinates system's polar angle. Two groups explicitly addressed this during construction of the $\hat{\boldsymbol{\alpha}}$ component.

Frank and Elliot invoke this symbolic form while constructing the radial component.

Frank: If you change $[\vec{M}]$ a little bit, $\alpha$, and, uh, $\beta$ doesn't change at all. This is just M because it's just the radius.

Here Frank is articulating that a differential length in the radial direction is independent of the angles and thus writes $d l_{M}=d M \hat{\mathbf{M}}$ without inscribing either angle into this component.

While the invocation of no dependence may seem trivial for a radial component, it played a larger role for Adam and Bart during the construction of the angular components.

Adam: For $\alpha$, it doesn't have any dependence on this other angle.

Here Adam recognizes and addresses that constructing the arc length term resulting from a change in $\alpha$ is independent of the angle $\beta$. As a result, students actively don't include a $\beta$ term in the component. For Adam, this meant that the $\hat{\boldsymbol{\beta}}$ component included an $\alpha$ term.

\section{CONCLUSIONS}

Application of the symbolic forms and concept image frameworks to student construction of a differential length vector enabled the identification of specific structures students associated with vector expressions as well as what concepts motivated these structures and the associated variables. Future work seeks to continue to integrate these frameworks further, to more completely account for students' integration of conceptual understanding with symbolic expression during differential length construction.
The explicit context of vectors and the increased mathematical sophistication of the upper-division content led to the identification of new forms in addition to symbolic forms previously identified. The symbol templates and associated schemata for the new differential and magnitude direction symbolic forms were consistent across groups, but the ideas motivating the invocation of the symbolic forms varied. For example, students often explained the need for the differential as having to account for a change or small amount of a quantity.

Further analysis identified that students at university A were able to recognize the general structure needed for the equation and invoke the correct template. The primary difficulties here constructing the differential length element were related to conceptual schemata. For example, students constructed an appropriate expression for the $\beta$ component in terms of dimensional and differential considerations, but the projection aspect of the concept image responsible for introducing the $\cos \alpha$ term was either misapplied or inaccessible. Students interviewed from University B were less successful invoking and combining necessary symbol templates and had difficulty accessing or applying ideas related to dimensionality or component and direction. Classroom observations at the first of the institutions suggest students are able to arrive at the general structure due to explicit and repeated emphasis on construction of differential length elements early in the semester. Even as such, students still are unable to connect the necessary ideas for differential length construction at this university. The exact nature of the difference in performance between the two universities is beyond the scope of this study, as we do not possess comparable data from classroom instruction at each site.

However, results do indicate instructional changes should focus on the concepts associated with the building of the differential, specifically making the explicit connection of the coefficients for the angle components to the idea of arc length and coordinate system geometry.

Dimensionality and geometric reasoning were especially prominent in the more successful efforts. In these cases, dimensionality and component and direction were closely tied, appearing when discussing overall structure and when isolating the change in each individual component. While reasoning about dimensionality and units was relevant to student construction, in some cases students struggled to determine the units of certain terms, such as angles and unit vectors. Findings suggesting the generalized use of units to expression construction are especially important as previous research on symbolic forms does not address how students' attention to units impacts their problem solving [12]. Geometric reasoning proved to be a more productive approach during construction. In many cases students attempted to visualize the paths traced by $\vec{M}$ as small changes were made to individual variables in the coordinate system. Most groups recognized the need for multiple components to properly express the differential length 
vector and appropriately connected the differentials to unit vectors of the same variable.

In cases where segments of construction proved difficult for students, recall mediated expression construction, similar to upper-division physical chemistry findings dealing with partial derivatives [27]. In our study, however, recall of spherical coordinates, despite having the potential to be productive, led students to construct expressions that incorrectly included a $\sin \alpha$ term. In several instances students attempted recall from Cartesian coordinates or projected $\vec{M}$ to the Cartesian axes. While this was in many cases only an attempt to understand the nature of the unconventional system, two groups explicitly constructed elements with Cartesian unit vectors. This continues to support the idea that students have more familiarity with Cartesian coordinates and have difficulty isolating ideas needed to construct differential vector elements in nonCartesian coordinate systems.

Subsequent efforts seek to report on students' understanding of differential volume elements and the connection to students' understanding of the length vector. A few groups recalled a spherical volume element in an attempt to reason about components during construction. More importantly, some students constructed the differential volume element from the terms in their length vector; the checking of a differential volume led these students to correct their initial mistakes. This has led to the development of student-centered instructional materials to be used in E\&M and/or mathematical methods of physics courses. In particular, we are developing and piloting a more scaffolded version of the interview task as a pair of instructional activities that guide students through the construction of components of the differential length vector, construction and checking of the volume element, and construction and choice of differential area vectors in schmerical coordinates.

\section{ACKNOWLEDGMENTS}

The authors would like to acknowledge Michael Loverude and Mikayla Mays for their part in organizing some of the logistical aspects of this study. This material is based upon work supported by the National Science Foundation under Grant No. PHY-1405726.
[1] L. Bollen, P. van Kampen, and M. De Cock, Students' difficulties with vector calculus in electrodynamics, Phys. Rev. ST Phys. Educ. Res. 11, 020129 (2015).

[2] L. Doughty, E. McLoughlin, and P. van Kampen, What integration cues, and what cues integration in electromagnetism, Am. J. Phys. 82, 1093 (2014).

[3] J. Guisasola, J. Almudí, J. Salinas, K. Zuza, and M. Ceberio, The Gauss and Ampere laws: Different laws but similar difficulties for student learning, Eur. J. Phys. 29, 1005 (2008).

[4] D. Hu and N. Sanjay Rebello, Using conceptual blending to describe how students use mathematical integrals in physics, Phys. Rev. ST Phys. Educ. Res. 9, 020118 (2013).

[5] R. E. Pepper, S. V. Chasteen, S. J. Pollock, and K. K. Perkins, Observations on student difficulties with mathematics in upper-division electricity and magnetism, Phys. Rev. ST Phys. Educ. Res. 8, 010111 (2012).

[6] T. I. Smith, J. R. Thompson, and D. B. Mountcastle, Student understanding of Taylor series expansions in statistical mechanics, Phys. Rev. ST Phys. Educ. Res. 9, 020110 (2013).

[7] J. Von Korff and N. Sanjay Rebello, Teaching integration with layers and representations: A case study, Phys. Rev. ST Phys. Educ. Res. 8, 010125 (2012).

[8] J. Wagner, Students' obstacles and resistance to Riemann sum interpretations of the definite integral, in Proceedings of the 19th Annual Conference on Research in Undergraduate Mathematics Education, Pittsburgh, Pennsylvania, edited by T. Fukawa-Connelly, N. Engelke Infante, M. Wawro, and S. Brown (Mathematical Association of America, Washington, DC, 2013).
[9] B. Wilcox, M. Caballero, D. Rehn, and S. Pollock, Analytic framework for students' use of mathematics in upper-division physics, Phys. Rev. ST Phys. Educ. Res. 9, 020119 (2013).

[10] T. Dray and C.A. Manogue, Using differentials to bridge the vector calculus gap, Coll. Math. J. 34, 283 (2003).

[11] D. Griffiths, Introduction to Electrodynamics, 4th ed. (Pearson Education, New York, 2013).

[12] B. Sherin, How students understand physics equations, Cognit. Instr. 19, 479 (2001).

[13] D. Tall and S. Vinner, Concept image and concept definition in mathematics with particular refernce to limits and continuity, Educ. Stud. Math. 12, 151 (1981).

[14] D. Hu and N. S. Rebello, Understanding student use of differentials in physics integration problems, Phys. Rev. ST Phys. Educ. Res. 9, 020108 (2013).

[15] D. Meredith and K. Marrongelle, How students use mathematical resources in an electrostatics context, Am. J. Phys. 76, 570 (2008).

[16] D. Nguyen and N. S. Rebello, Students' difficulties with integration in electricity, Phys. Rev. ST Phys. Educ. Res. 7, 010113 (2011).

[17] M. Artigue, J. Menigaux, and L. Viennot, Some aspects of students' conceptions and difficulties about differentials, Eur. J. Phys. 11, 262 (1990).

[18] S. Jones, Understanding the integral: Students' symbolic forms, J. Math. Behav. 32, 122 (2013). 
[19] C. Manogue, K. Browne, T. Dray, and B. Edwards, Why is Ampère's law so hard? A look at middle-division physics, Am. J. Phys. 74, 344 (2006).

[20] C. Singh, Student understanding of symmetry and Gauss' law of electricity, Am. J. Phys. 74, 923 (2006).

[21] C. Wallace and S. Chasteen, Upper-division students' difficulties with Ampère's law, Phys. Rev. ST Phys. Educ. Res. 6, 020115 (2010).

[22] B. E. Hinrichs, Writing position vectors in 3-d space: A student difficulty with spherical unit vectors in intermediate E\&M, AIP Conf. Proc. 1289, 173 (2010).

[23] E. Sayre and M. Wittmann, Plasticity of intermediate mechanics students' coordinate system choice, Phys. Rev. ST Phys. Educ. Res. 4, 020105 (2008).

[24] M. Vega, W. Christensen, B. Farlow, G. Passante, and M. Loverude, Student understanding of unit vectors and coordinate systems beyond cartesian coordinates in upper division physics courses, Proceedings of the Physics Education Research Conference, Sacramento, CA, 2016, https://www.compadre.org/Repository/document/ServeFile .cfm?ID=14270\&DocID=4624.

[25] T. Paoletti, K. C. Moore, J. Gammaro, and S. Musgrave, Students' emerging understanding of the polar coordinate system, Proceedings of the 16th Annual Conference on Research in Undergraduate Mathematics Education, Denver, Colorado, edited by S. Brown, G. Karakok, K. H. Roh, and M. Oehrtman (Mathematical Association of America, Washington, DC, 2013).

[26] A. A. DiSessa, Toward an Epistemology of Physics, Cognit. Instr. 10, 105 (1993).

[27] N. Becker and M. Towns, Students' understanding of mathematical expressions in physical chemistry contexts: An analysis using Sherin's symbolic forms, Chem. Educ. Res. Pract. 13, 209 (2012).

[28] P. Heron, Empirical investigations of learning and teaching, part I: Examing and interpreting student thinking, in Enrico Fermi Summer School on Physics Education Research, edited by E. F. Redish and M. Vincentini (Italian Physical Society, Varenna, Italy, 2003), pp. 341-351.

[29] D. Roundy, C. Manogue, J. Wagner, E. Weber, and T. Dray, An extended theoretical framework for the concept of derivative, in Proceedings of the 18th Annual Conference on Research in Undergraduate Mathematics Education, Pittsburgh, Pennsylvania, edited by T. Fukawa-Connelly, N. Infante, K. Keene, and M. Zandieh (Mathematical Association of America, Washington, DC, 2015). 